\title{
Surface imaging of stellar non-radial pulsations
}

\section{The $\beta$ Cephei star $\omega^{1}$ Sco ${ }^{\star}$}

\author{
S. V. Berdyugina ${ }^{1,2}$, J. H. Telting ${ }^{3}$, H. Korhonen ${ }^{1,4}$, and C. Schrijvers ${ }^{5}$ \\ 1 Astronomy Division, University of Oulu, PO Box 3000, 90401 Oulu, Finland \\ 2 Institute of Astronomy, ETHZ, 8092 Zurich, Switzerland \\ 3 Nordic Optical Telescope, Apartado 474, 38700 S/C de La Palma, Canarias, Spain \\ 4 Astrophysical Institute Potsdam, An der Sternwarte 16, 14482 Potsdam, Germany \\ 5 Astronomical Institute Anton Pannekoek, University of Amsterdam, Kruislaan 403, 1098 SJ Amsterdam, The Netherlands
}

Received 10 February 2003 / Accepted 13 May 2003

\begin{abstract}
We apply the surface imaging technique to high-resolution spectra of the rapidly rotating $\beta$ Cep-type star $\omega^{1}$ Sco which shows strong line-profile variations in the Si III $\lambda$ 4552, 4567, $4574 \AA$ triplet. These variations have been interpreted in terms of non-radial pulsations by Telting \& Schrijvers (1998). Their analysis of the spectral time series with traditional Fourier transforms resulted in one significant frequency, suggesting that the profile variations are due to only one dominant pulsation mode. Here we apply a spectral inversion technique to the same time series of the profiles. Assuming only temperature fluctuations due to pulsations, we obtain an image of the dominant pulsation mode. We estimate the pulsation degree from the image, put constraints on the azimuthal number of the mode, and study the latitudinal distribution of the pulsation pattern. In addition to the known dominant mode we find traces of a second pulsation mode.
\end{abstract}

Key words. stars: oscillations - stars: imaging - stars: early-type - stars: individual: $\omega^{1}$ Sco

\section{Introduction}

In the early 1980 s, systematic line-profile variations $(l p v)$ were discovered in a number of early-type stars. The lpv take the form of "bumps" which cross the absorption lines on time scales of hours. A pioneer study by Vogt \& Penrod (1983) showed that the crossing times of the features are too rapid to be caused by stellar rotation. They argued that the $l p v$ arise in photospheric non-radial pulsations (NRP) which re-distribute the flux in a rotationally broadened profile due to combined effects of the pulsational velocity field and temperature fluctuations.

The phenomenon of such $l p v$ due to NRP is found to be widespread among early-type stars. The mode-identification problem can be solved with different methods (see Telting 2003 for a recent review). In the first paper of the present series (Berdyugina et al. 2003, hereafter Paper I), we showed that the traditional stellar surface imaging technique can provide useful information on the dominant pulsation mode. We performed inversions of simulated data and found that tesseral modes with $\ell-|m|=2$ as well as sectoral modes can be successfully recovered under various conditions. Tesseral modes with $\ell-|m|=1$ (or any odd number) appeared rather unfortunate in the surface maps, especially at higher inclination of the stellar rotation axis, as they produce very small $l p v$. At lower inclinations, the

Send offprint requests to: S. V. Berdyugina,

e-mail: sveta@astro.phys.ethz.ch

* Based on observations collected at ESO, La Silla. $l p v$ are larger, and the azimuthal order $|m|$ can be successfully recovered.

Here we present results of the first attempt to map surface fluctuations of the rapidly rotating $\beta$ Cephei star $\omega^{1}$ Sco (HD 144470, B1 V) with the stellar surface imaging technique. $\omega^{1}$ Sco was discovered by Telting \& Schrijvers (1998, hereafter TS98) as a rapidly rotating $\beta$ Cephei star showing remarkable line-profile variations in the Si III triplet. They interpreted the $l p v$ in terms of non-radial pulsations and determined a single significant frequency, 15 cycles/day, using traditional Fourier transforms. This suggested that the profile variations are due to one dominant pulsation mode. A pulsational degree of the mode, $\ell=9 \pm 1$, was determined as well. A value of the azimuthal number $m$ could not be determined from the profiles, although it was suggested that the mode is prograde and sectoral, i.e. $m=-9$. In the present paper, we apply the surface imaging inversion technique to the time series of profiles obtained by TS98. Our study confirms the earlier conclusion on the dominant pulsating mode and reveals a trace of the second mode, which modulates the dominant one.

\section{Inversions}

\subsection{The data}

The observations have been published by TS98 where one can find detailed description of the data and reducing procedure. 
The spectra with resolution $R=65000$ were obtained at ESO, La Silla in 1996 and covered the wavelength region of the Si III triplet at $\lambda$ 4552, 4567, $4574 \AA$ and have a $S / N$ ratio typically between 500 and 1000 . A typical exposure time was $15 \mathrm{~min}$.

\subsection{The technique}

For the sake of simplicity, we assume that the $l p v$ are caused by temperature fluctuations only. In Paper I we showed that for stars with rotationally broadened line profiles the mode identification is not sensitive to such an assumption, and that the resulting maps can aid significantly in mode identification.

A line list was compiled with the Vienna database VALD (Kupka et al. 1999). It included the Si III triplet and blending lines stronger than $1 \%$ in absorption at temperature $25000 \mathrm{~K}$. We noticed that using only one line of the triplet in inversions produces noisy images, while simultaneous inversion of the three lines reduce the noise remarkably. Therefore, all presented results were obtained with the three lines.

A stellar image is considered as the distribution of effective temperatures of the stellar models across the stellar surface. Using stellar atmosphere models by Kurucz (1993), we calculated a set of local line profiles for the temperature range of $20000-30000 \mathrm{~K}$ and $\log g=4.0$. The surface of the star was divided by the coordinate grid of $3^{\circ} \times 3^{\circ}$, and the temperature of each pixel was recovered in the inversion. The effect of displacement of the pixels during the exposure time was not taken into account.

As well as in Paper I, we used the Occamian approach as the inversion technique (Berdyugina 1998). Our technique is free from assumptions that pulsations cause line profile variations. In contrast with many other techniques, we do not assume that the surface-feature distribution is in the form of spherical harmonics or any other presupposed shape, when we do the inversion. This provides an advantage to test if such an assumption made in previous studies is reliable. Also, we do not make any assumptions if pulsation modes are of sectoral or tesseral type. Our imaging technique is used to reveal this from the data.

\subsection{Stellar parameters}

A number of stellar parameters have to be fixed in the inversions, such as the inclination of the rotation axis to the line of sight $i$, rotational velocity $v \sin i$, and pattern-rotation period $P_{\mathrm{r}}$. In Paper I we studied the influence of errors in these parameters on inversion results. We found that variations in the first two parameters do not disturb significantly the pulsation pattern and, thus, do not influence the mode identification. Errors in the pattern-rotation period appeared however to be critical for inversions.

The most critical parameter, the pattern-rotation period, can be estimated from the observed frequency. TS98 found that the dominant pulsation mode oscillates with the frequency of 15.0 cycles/day and is probably associated with the mode $|m|=9$, if the inclination angle is high. For this mode the pattern-rotation period $P_{\mathrm{r}}$ value would be 14.4 . However, it was also noticed that at lower inclination angles a mode $|m|=8$ is
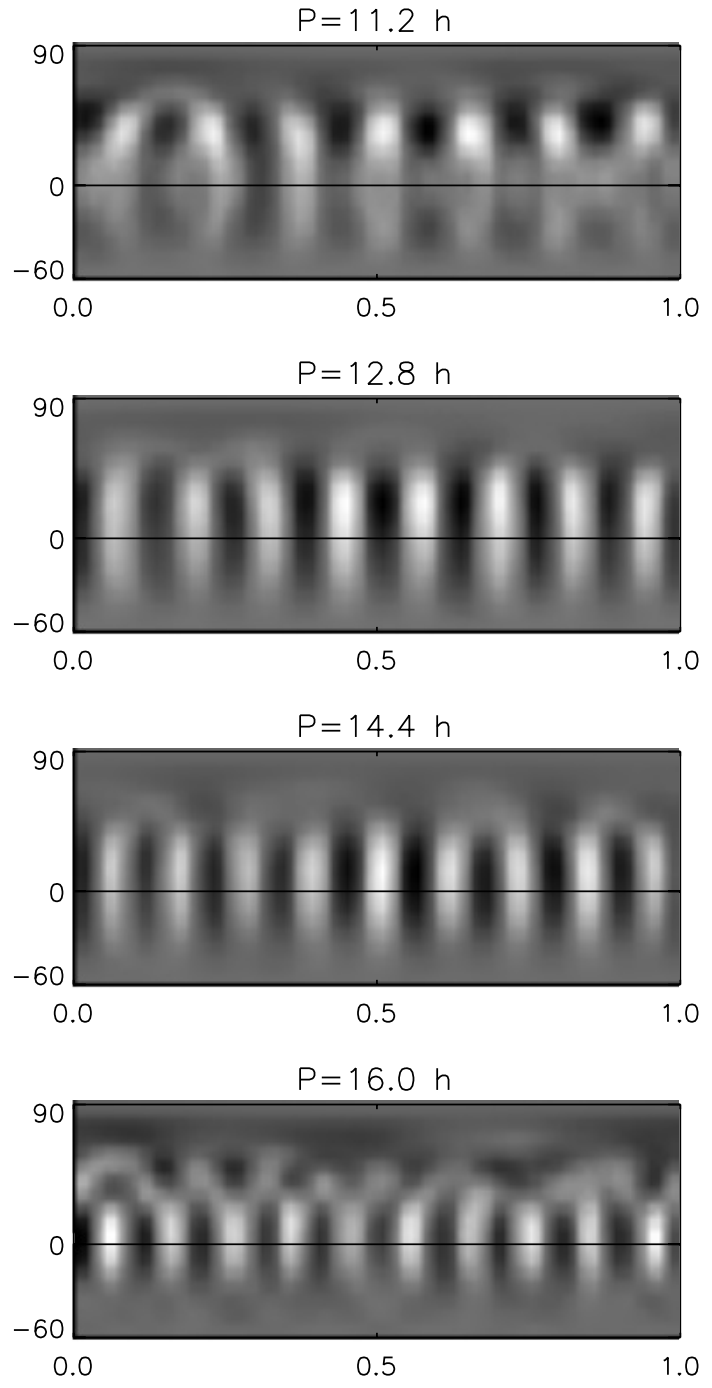

Fig. 1. Images recovered with different values of the pattern-rotation period for the inclination angle of $60^{\circ}$ and rotational velocity $100 \mathrm{~km} \mathrm{~s}^{-1}$. The periods $11^{\mathrm{h}} .2,12^{\mathrm{h}} .8,14^{\mathrm{h}} .4$ and $16^{\mathrm{h}} .0$ correspond to the pulsational modes $|m|=7,8,9,10$, respectively.

also appropriate. The $P_{\mathrm{r}}$ value for this mode would be 12 h. 8 . We carried out inversions for both periods at intermediate inclination $i=60^{\circ}$ and found that they indeed result in clear images of sectoral pulsation patterns with $|m|=8$ and $|m|=9$ (Fig. 1), suggesting that either of the two periods can be resonant. For shorter and longer periods, for instance for 11.2 and $16^{\mathrm{h}} .0$ which would correspond to the modes $|m|=7$ and $|m|=10$, respectively, the images do not show clear pulsation patterns (Fig. 1). This suggests that the dominant pulsational mode is indeed of sectoral or near-sectoral type. We further discuss the mode-identification in Sect. 3. Small deviations from the resonant periods results in smearing of the pulsation pattern that does not affect the mode-identification (see Fig. 2). Since the images for both resonant periods are about the same quality, we test other stellar parameters only for one of them.

The rotational velocity of $\omega^{1}$ Sco was determined by Slettebak et al. (1975) and Brown \& Verschueren (1997): $110 \mathrm{~km} \mathrm{~s}^{-1}$ and $100 \mathrm{~km} \mathrm{~s}^{-1}$, respectively. We carried out calculations for these two values and for the mid value $105 \mathrm{~km} \mathrm{~s}^{-1}$ 

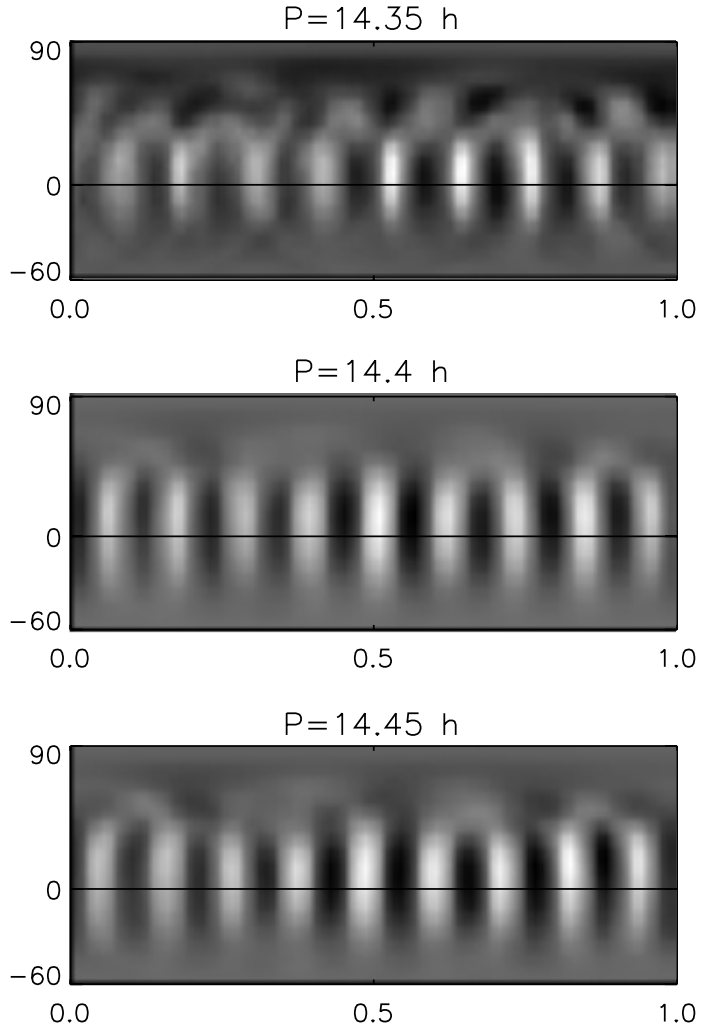

Fig. 2. Images recovered with small variations $\left( \pm 0^{\natural} \cdot 05\right)$ of the patternrotation period for the inclination angle of $60^{\circ}$ and rotational velocity $100 \mathrm{~km} \mathrm{~s}^{-1}$.

(Fig. 3). In Paper I we found that underestimating $v \sin i$ results in darkening of the polar region, while overestimating of $v \sin i$ results in its brightening. Therefore, we conclude that for $\omega^{1} \operatorname{Sco} v \sin i=105 \mathrm{~km} \mathrm{~s}^{-1}$ is more appropriate.

The inclination of the rotational axis to the line of sight is difficult to determine from observations. In the previous study of the star by TS98 it was found that the inclination of the star should be larger than $20^{\circ}$. Since this parameter is not critical for mode identification, we can assume, for instance, that $i=60^{\circ}$ and check if other values would be more appropriate. In Fig. 4 results for three inclination angles are presented. In a good agreement with the results of Paper I higher angles are preferable, although this parameter remains uncertain.

\subsection{Results}

A recovered image of $\omega^{1}$ Sco and fits to the observed profiles obtained for an optimal set of stellar parameters: $P_{\mathrm{r}}=14.4$, $i=60^{\circ}, v \sin i=105 \mathrm{~km} \mathrm{~s}^{-1}$ are shown in Fig. 5. A clear sectoral mode of $\ell=|m|=9$ is read from the image. The temperature fluctuations are concentrated in the equatorial zone, in good agreement with predictions of sectoral NRP.

Although the resulted temperature range of the map is large, only few pixels in the map take the extreme values. The average temperature of bright and dark regions in the map are of $28300 \mathrm{~K}$ and $22600 \mathrm{~K}$, respectively. The temperature fluctuations $\Delta T / T$ are within $\pm 10 \%$ of the effective temperature. This results in the fit of the line profiles with $S / N=360$,
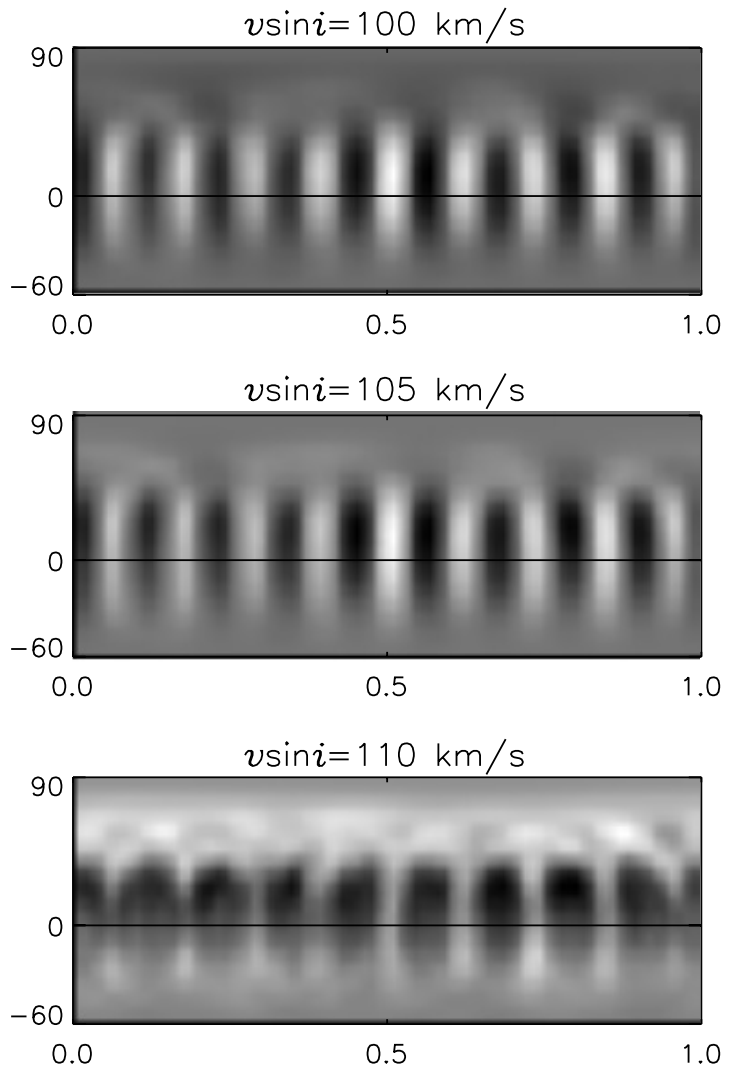

Fig. 3. Images recovered with three values of the rotational velocity for the inclination angle $60^{\circ}$ and pattern-rotation period 14.4 .
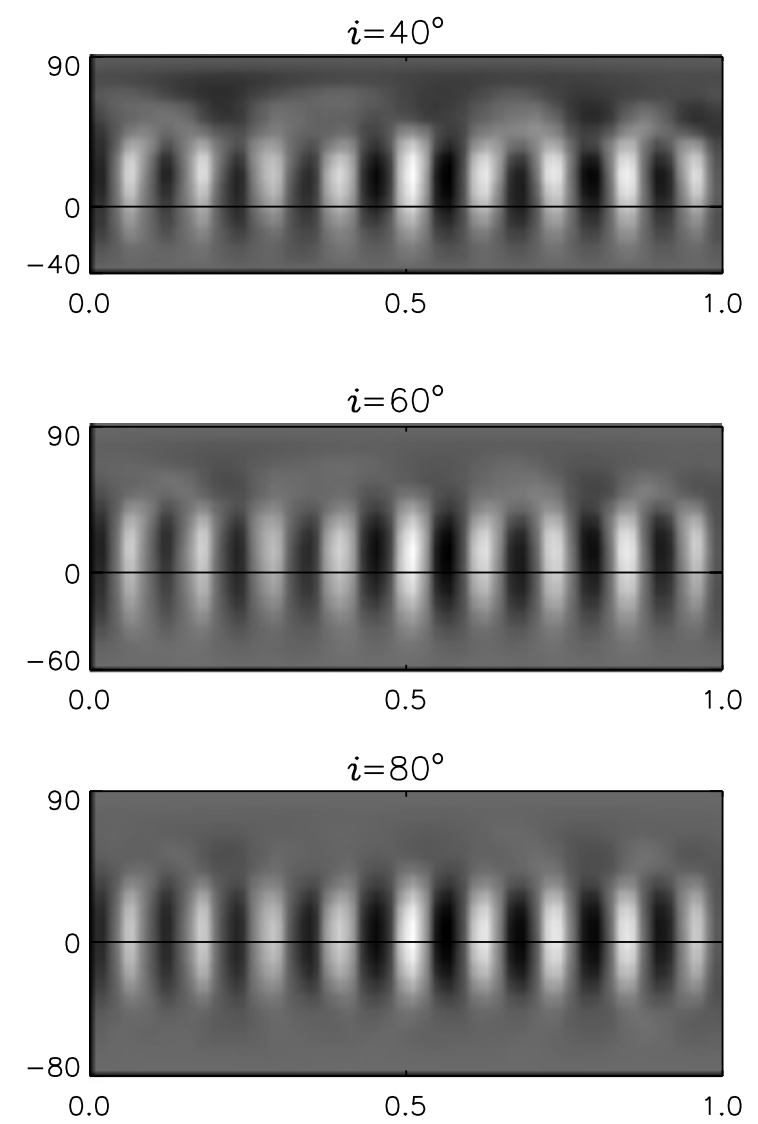

Fig. 4. Images recovered with three values of the inclination angles for the rotational velocity $105 \mathrm{~km} \mathrm{~s}^{-1}$ and pattern-rotation period 14.4. 

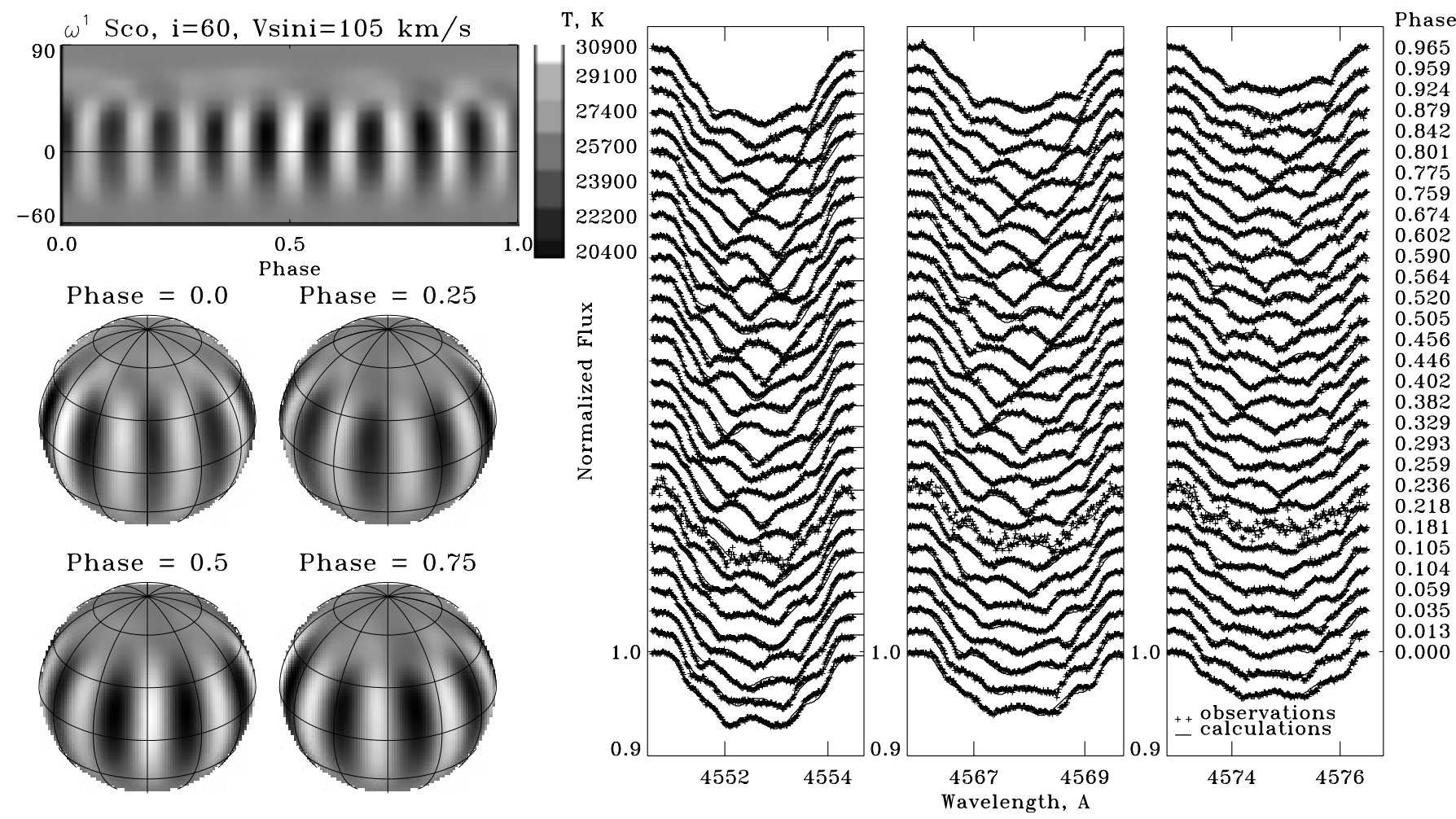

Fig. 5. The image of $\omega^{1}$ Sco, recovered under the assumption of only temperature variations, and fits to the observed line profiles. The image is shown in the cylindrical and spherical projections.

although the observed $S / N$ ratios were typically between 500 and 1000. Small discrepancies in the fits of the profiles suggest for the presence of the velocity field fluctuations which have been ignored. This is also confirmed by the fact that cooler regions in the image are slightly broader than the hotter ones. As we noticed in Paper I, this is an indication of the presence of significant velocity fluctuations. Modeling the line profile variations with NRP by TS98 resulted in estimates of the surface velocity amplitude of at least $10 \mathrm{~km} \mathrm{~s}^{-1}$, provided that the surface temperature variations are small. If this is interpreted in temperature fluctuations, then $\Delta T / T=10 \%$ is rather appropriate (TS98, Paper I).

\section{Discussion}

We can couple the results of the surface maps to the findings of TS98, who derived a constraint for the degree of the dominant mode in $\omega^{1}$ Sco: $\ell=9 \pm 1$. From Fig. 1 and the constraint on $\ell$ we find the following:

- $|m|=10=\ell$ can be ruled out as the map for the patternrotation period of $P=16.0$ rules out a sectoral mode (Fig. 1);

- $|m|=9=\ell$ and $|m|=8=\ell$ give consistent and clear maps with sectoral modes (Figs. 1 and 5);

- $|m|=8$ with $\ell=9$ or $\ell=10$ is not a possible solution, because, as we have shown in Paper I, in such cases pulsation bumps should show much more contrast at higher latitudes, while in our map for a pattern-rotation period of $P=12$ h. 8 they are very close to the equator (Fig. 1);
- $|m|=7$ with $\ell=8$ is possible (upper panel in Fig. 1), because, as we have seen in Paper I, bumps of such a mode have high contrast in higher latitudes, and this map seems to show a reflection pattern that was seen for modes with $\ell-|m|=1$ in Paper I;

- $|m|=7$ with $\ell=9$ is not favorable, because all our calculations for $\ell-|m|=2$ modes show a clear equatorial pattern which is absent in the map with $P=11$. 2 .

After this consideration, we conclude that possible modes are $\ell=9$ with $|m|=9$, and $\ell=8$ with $|m|=8$ or $|m|=7$.

We note that for a non-sectoral mode at high inclination to give such large line-profile variations as observed in $\omega^{1} \mathrm{Sco}$, we expect the mode to have a very high amplitude. Indeed, in our map for the pattern-rotation period of 11.2 hours, which is consistent with an $\ell=8$ and $|m|=7$ mode, we find that the amplitude of the variations is 1.3 times higher than for the maps showing sectoral modes (see Fig. 1).

An inspection of the profiles and the map (Fig. 5) reveals different amplitudes of the pulsations in the opposite stellar hemispheres divided approximately by the meridians of $145^{\circ}$ and $325^{\circ}$ corresponding to phases 0.4 and 0.9 . In the hemisphere from $145^{\circ}$ to $325^{\circ}$, the amplitude of the pulsations is larger than in the opposite hemisphere, from $325^{\circ}$ to $145^{\circ}$ (Fig. 6). In temperature variations, this difference is about $2500 \mathrm{~K}$, that is comparable with the recovered amplitude of the pulsations and, thus, significantly larger than the errors of the map. Assuming that the profile variations are due a sectoral $\ell=9$ mode (as found in Fig. 5), the amplitude modulation in the opposite hemispheres of the star suggests for the presence 


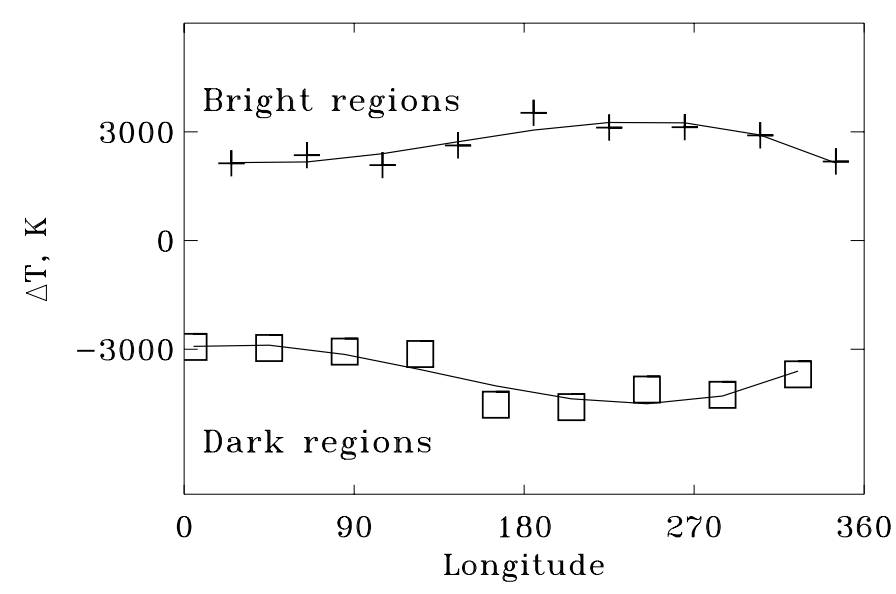

Fig. 6. Amplitudes of the pulsation "bumps" measured in temperatures from the image presented in Fig. 5. The variations suggest for the presence of the second pulsation mode with $\ell$ close to the dominant mode. It is identified as a mode of $\ell=8$ and the frequency of 13.33 cycles/day.

of another sectoral mode with $|m|$ close to that of the dominant mode. This produces a "beating" pattern, so that on one side of the star the amplitudes add constructively, and on the other side they cancel out. A mode with $|m|=8$ could be appropriate, and should have a frequency close to that of the dominant mode. We note that a low-power frequency peak at 13.6 cycles/day was found in the data in TS98, which also indicates that there may be more than one pulsation mode in $\omega^{1}$ Sco. We stress that the relatively small number of spectra that we have analysed do not suffice to make conclusive statements regarding the frequency of a possible second mode.

As seen from Fig. 6, the temperature fluctuations due to pulsations perfectly compensate each other and produce no large-scale effect when summed over the stellar surface. Also, the flux in the continuum calculated for the temperature distribution presented in Fig. 5 does no show significant variations. This result is supported by the fact that $\omega^{1}$ Sco shows remarkable photometric stability and has served as the primary standard star of the Walraven photometric system (Lub $\&$ Pel 1977). In the Hipparcos catalogue, $\omega^{1}$ Sco is marked as constant with a standard error of $0.7 \mathrm{mmag}$. To further constrain the photometric variability of $\omega^{1}$ Sco, the velocity field fluctuations should be taken into account in the inversions.
This would certainly reduce the amplitudes of the temperature fluctuations that we derive.

\section{Conclusions}

We showed that the traditional technique of imaging of stellar surfaces can be successfully used for mapping stellar nonradial pulsations in terms of temperature fluctuations. The technique results in an image of the stellar surface in which sectoral and tesseral modes can be distinguished. In case of multiple mode NRP, it provides additional information and helps to identify low-power modes.

The surface of the rapidly rotating B1 V star $\omega^{1}$ Sco was mapped under the assumption that the observed line-profile variations are due to only temperature fluctuations. No assumption about the origin of the temperature fluctuations was made. The Occamian approach was used as an imaging technique. The distribution of the temperature fluctuations in the dominant pulsation mode ( 15 cycles/day) clearly suggests the presence of non-radial pulsations. The pulsations were found to be concentrated to the equator in good agreement with a sectoral mode of $\ell=|m|=9$ or $\ell=|m|=8$; the tesseral mode of $\ell=8$ with $|m|=7$ is also consistent with one of the investigated surface maps.

The surface map of the dominant pulsational mode shows amplitude modulation in the opposite hemispheres of the star. This suggests the presence of another sectoral mode with $\ell \sim 8$ with a frequency close to that of the dominant mode.

\section{References}

Berdyugina, S. V. 1998, A\&A, 338, 97

Berdyugina, S. V., Telting, J. H., \& Korhonen, H. 2003, A\&A, 406, 273 (Paper I)

Brown, A. G. A., \& Verschueren, W. 1997, A\&A, 319, 811

Kupka, F., Piskunov, N., Ryabchikova, T. A., Stempels, H. C., \& Weiss, W. W. 1999, A\&AS, 138, 119

Kurucz, R. L. 1993, Kurucz CD No. 13

Lub, J., \& Pel, J. W. 1977, A\&A, 54, 137

Slettebak, A., Collins, G. W., Boyce, P. B., White, N. M., \& Parkinson T. D. 1975, ApJS, 29, 137

Telting, J. H. 2003, in Asteroseismology Across the HR Diagram, ed. M. J. Thompson, M. S. Cunha, \& M. J. P. F. G. Monteiro (Kluwer) Telting, J. H., \& Schrijvers, C. 1998, A\&A, 339, 150 (TS98)

Vogt, S. S., \& Penrod, G. D. 1983, ApJ, 275, 661 